\title{
Neutrino-nucleus reactions based on new shell model Hamiltonians
}

\author{
Toshio Suzuki* \\ Department of Physics, College of Humanities and Sciences, Nihon University, Sakurajosui 3-25-40, Setagaya-ku, Tokyo 156-8550, Japan
}

Satoshi Chiba

Advanced Science Research Center, Japan Atomic Energy Agency, 2-4 Shirakata-shirane, Tokai, Naka-gun, Ibaraki 319-1195, Japan

Takashi Yoshida

National Astronomical Observatory of Japan, Mitaka, Tokyo 181-8588, Japan

Toshitaka Kajino

National Astronomical Observatory of Japan, Mitaka, Tokyo 181-8588, Japan

and Department of Astronomy, Graduate School of Science, University of Tokyo, Bunkyo-ku, Tokyo 113-0033, Japan

Takaharu Otsuka

Department of Physics and Center for Nuclear Study, University of Tokyo, Hongo, Bunkyo-ku, Tokyo 113-0033, Japan and RIKEN, Hirosawa, Wako-shi, Saitama 351-0198, Japan

(Received 29 May 2006; published 6 September 2006)

\begin{abstract}
A new shell model Hamiltonian for $p$-shell nuclei which properly takes into account important roles of spin-isospin interactions is used to obtain cross sections of neutrino- ${ }^{12} \mathrm{C}$ reactions induced by decay-at-rest neutrinos as well as supernova neutrinos. Branching ratios to various decay channels are calculated by the Hauser-Feshbach theory. Neutrino- ${ }^{4} \mathrm{He}$ reactions are also investigated by using recent shell model Hamiltonians. The reaction cross sections are found to be enhanced for both ${ }^{12} \mathrm{C}$ and ${ }^{4} \mathrm{He}$ compared with previous calculations. As an interesting consequence of this, a possible enhancement of the production yields of light elements, ${ }^{7} \mathrm{Li}$ and ${ }^{11} \mathrm{~B}$, during supernova explosions is pointed out.
\end{abstract}

DOI: 10.1103/PhysRevC.74.034307

PACS number(s): 25.30.-c, 21.60.Cs

\section{INTRODUCTION}

A recent progress in shell model calculations, which properly takes into account important roles of spin-isospin interactions, is found to lead to significant improvements in magnetic properties of nuclei [1] as well as proper shell evolution, that is, the change of magic numbers toward the drip lines [2,3]. An important general role of the tensor interaction is pointed out [3]. The modified Hamiltonian can explain spin properties of the $p$-shell nuclei such as Gamow-Teller transitions better than conventional shell model Hamiltonians. In particular, agreements between calculated and observed magnetic moments are found to be systematically improved for the $p$-shell nuclei [1].

Here, we study new ingredients of these developments on neutrino-nucleus reactions on ${ }^{12} \mathrm{C}$, which are dominantly induced by Gamow-Teller and spin-dipole transitions. Chargeexchange and neutral current reactions induced by neutrinos from pion decay-at-rest (DAR) and supernova neutrinos are investigated, and comparisons are made with previous calculations [4-6]. We also study neutrino-nucleus recations on ${ }^{4} \mathrm{He}$, which are mainly induced by spin-dipole transitions. We discuss possible effects of our new neutrino-nucleus reaction cross sections on the nucleosynthesis process, in particular, on light-element abundances during supernova explosions.
The paper is organized as follows. In Sec. II, we discuss tensor components of our modified interaction. Neutrinonucleus reaction cross sections on ${ }^{12} \mathrm{C}$ and ${ }^{4} \mathrm{He}$ are obtained by using our new shell model Hamiltonian in Sec. III. Astrophysical implications are discussed in Sec. IV, and a summary is given in Sec. V.

\section{NEW SHELL MODEL HAMILTONIAN FOR p-SHELL NUCLEI}

We discuss some important ingredients of our new shell model Hamiltonian for $p$-shell nuclei in Ref. [1], where the spin-isospin flip interaction and the shell gap between the $0 p_{1 / 2}$ and $0 p_{3 / 2}$ orbits are enhanced in comparison to those obtained by the Cohen-Kurath Hamiltonian (8-16)2BME [7]. We will refer to this as the SFO Hamiltonian hereafter.

First, we show that the dominant effect of the enhancement of the spin-flip two-body matrix elements in the isospin $T=0$ channel, $\left\langle 0 p_{3 / 2} 0 p_{1 / 2}: J T|V| 0 p_{3 / 2} 0 p_{1 / 2}: J T\right\rangle$ with $J=1,2$, is the modification of the tensor component of the interaction. Spin-tensor decomposition of the two-body effective interactions for a specific isospin channel can be done by expanding the matrix element with those of the same orbital angular momenta $[8,9]$. Each matrix element is decomposed into the central $(k=0)$, spin-orbit $(k=1)$, and tensor $(k=2)$ components,

$$
\langle a b: J T|V| c d: J T\rangle=\sum_{k}\left\langle a b: J T\left|V_{k}\right| c d: J T\right\rangle,
$$

*Electronic address: suzuki@chs.nihon-u.ac.jp 
with

$$
\begin{aligned}
& \left\langle a b: J T\left|V_{k}\right| c d: J T\right\rangle=(-1)^{J}(2 k+1) \\
& \quad \times \sum_{L L^{\prime} S S^{\prime}}\langle a b \mid L S J\rangle\left\langle c d \mid L^{\prime} S^{\prime} J\right\rangle\left\{\begin{array}{ccc}
L & S & J \\
S^{\prime} & L^{\prime} & k
\end{array}\right\} \\
& \times \sum_{J^{\prime}}(-1)^{J^{\prime}}\left(2 J^{\prime}+1\right)\left\{\begin{array}{ccc}
L & S & J^{\prime} \\
S^{\prime} & L^{\prime} & k
\end{array}\right\} \\
& \times \sum_{j_{a}^{\prime} j_{b}^{\prime} j_{c^{\prime}}^{\prime} j_{d}^{\prime}}\left\langle a^{\prime} b^{\prime} \mid L S J^{\prime}\right\rangle\left\langle c^{\prime} d^{\prime} \mid L^{\prime} S^{\prime} J^{\prime}\right\rangle \\
& \times\left\langle a^{\prime} b^{\prime}: J^{\prime} T|V| c^{\prime} d^{\prime}: J^{\prime} T\right\rangle,
\end{aligned}
$$

where $a=\left\{n_{a} \ell_{a} j_{a}\right\}, a^{\prime}=\left\{n_{a} \ell_{a} j_{a}^{\prime}\right\}$, and

$$
\langle a b \mid L S J\rangle=\left\{\begin{array}{ccc}
\ell_{a} & 1 / 2 & j_{a} \\
\ell_{b} & 1 / 2 & j_{b} \\
L & S & J
\end{array}\right\} \hat{j_{a}} \hat{j_{b}} \hat{L} \hat{S}
$$

with $\hat{L}=2 L+1$, etc.

The monopole terms of the three components

$$
V_{k}^{j_{1} j_{2}, M}=\frac{\sum_{J}(2 J+1)\left\langle j_{1} j_{2}: J T\left|V_{k}\right| j_{1} j_{2}: J T\right\rangle}{\sum_{J}(2 J+1)}
$$

are shown in Fig. 1(a) for the $p$-shell matrix elements with $T=$ 0 for the SFO and the original Cohen-Kurath Hamiltonian. We find that the most important modification appears in the tensor components, for which even the signs of the matrix elements are changed. The central components of the monopole terms are also increased. Note that the total $p$-shell matrix elements are renormalized by a factor of 0.93 [1].

The attractive (repulsive) nature of the tensor components of the monopole matrix elements with $j_{1}=j_{>}=0 p_{3 / 2}$ and $j_{2}=j_{<}=0 p_{1 / 2}\left(j_{1}=j_{2}=j_{>}=0 p_{3 / 2}\right.$ or $j_{1}=j_{2}=j_{<}=$ $\left.0 p_{1 / 2}\right)$ is consistent with the general robust nature of the tensor interaction [3].

Although the magnitude of the tensor components of the monopole matrix elements of the SFO Hamiltonian is small compared with that of the pion and $\rho$-meson $(\pi+\rho)$ exchange potential with a radial cutoff at $0.7 \mathrm{fm}[10]$ and the M3Y interaction [11], their signs and the zigzag pattern of the (a) Monopole matrix elements

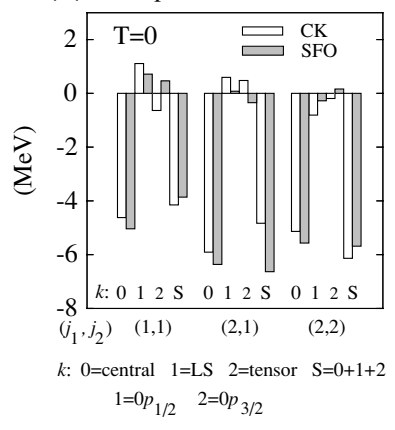

(b) Monopole matrix elements

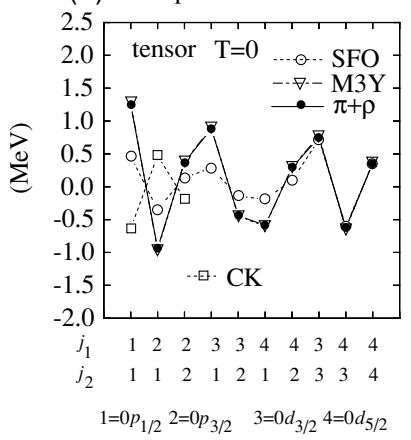

FIG. 1. (a) Monopole terms of the central $(k=0)$, spin-orbit $(k=$ $1)$, and tensor $(k=2)$ components of the SFO and Cohen-Kurath interactions. (b) Monopole terms of the tensor component of the SFO and Cohen-Kurath interactions as well as the $\pi+\rho$ exchange potential and the M3Y interaction.

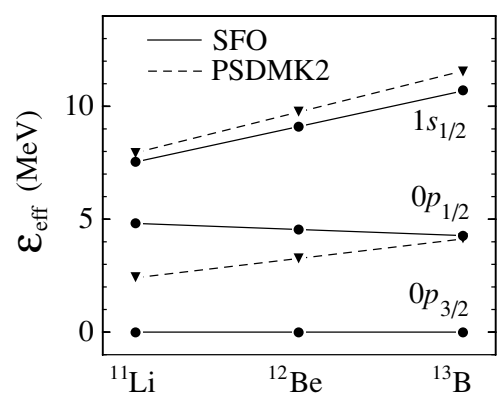

FIG. 2. Effective neutron single-particle energies relative to that of the $0 p_{3 / 2}$ orbit for $N=8$ isotones.

monopole matrix element as a function of $j_{>}-j_{<}$and $j_{>}-j_{>}$ or $j_{<}-j_{<}$are consistent as shown in Fig. 1(b). This zigzag structure with the proper signs in the tensor monopole terms is important and essential for the proper shell evolution.

Next, we show how these important characteristics affect the behavior of the effective single-particle energies. Effective neutron single-particle energies for $N=8$ isotones are shown in Fig. 2. The effective single-particle energy is the sum of the bare single-particle energy for the ${ }^{4} \mathrm{He}$ core and monopole two-body matrix elements of the proton-neutron $(p-n)$ interaction summed over occupied proton orbits outside the ${ }^{4} \mathrm{He}$ core. Since the tensor interaction is attractive between the proton $(\pi) 0 p_{3 / 2}$ orbit and neutron $(v) 0 p_{1 / 2}$ orbit while it is repulsive between the $\pi 0 p_{3 / 2}$ and $\nu 0 p_{3 / 2}$ orbits, the energy gap between the $\nu 0 p_{1 / 2}$ and $\nu 0 p_{3 / 2}$ orbits becomes larger as the proton number gets smaller, that is, for more neutron-rich isotones. The monopole terms of the central interaction are attractive both for $j_{1}=\pi 0 p_{3 / 2}, j_{2}=\nu 0 p_{1 / 2}$ and $j_{1}=\pi 0 p_{3 / 2}, j_{2}=\nu 0 p_{3 / 2}$, and their difference has the same sign as the tensor interaction, but the magnitude is smaller by about half. The monopole terms of the spin-orbit interaction work opposite to the tensor and central interactions. The proper shell evolution is not obtained in the case of the original Cohen-Kurath interaction as the monopole terms of the tensor components have opposite signs compared to the SFO interaction, which results in the reduction of the energy gap between the $\nu 0 p_{1 / 2}$ and $\nu 0 p_{3 / 2}$ orbits in the neutron-rich side.

We shall now go on to the question of the extent to which such a shell evolution is related to neutrino-nucleus reactions.

\section{NEUTRINO-NUCLEUS REACTIONS}

\section{A. Reactions on ${ }^{12} \mathrm{C}$ induced by DAR neutrinos}

We showed in Ref. [1] that the magnetic properties of $p$-shell nuclei are considerably improved, for example, in magnetic moments and Gamow-Teller transitions. Here, we study another example of spin-dependent transitions, namely, neutrino-nucleus reactions, which are induced mainly by excitations of Gamow-Teller and spin-dipole states.

We focus here on reactions on ${ }^{12} \mathrm{C}$, as the Gamow-Teller transition to the ground states of ${ }^{12} \mathrm{~N}$ and ${ }^{12} \mathrm{~B}$ have been studied quite well $[1,12]$. Charge-exchange reactions as well as neutral current reactions induced by DAR neutrinos are investigated. 
The electron neutrinos produced from the DAR pions and the $\mu^{+}$decay have an average energy of about $35 \mathrm{MeV}$ with an upper limit at $52.8 \mathrm{MeV}$. The reactions are induced predominantly by the axial-vector current. Contributions from the vector current are rather small but not negligible.

The multipole expansions of the reaction cross sections induced by $v$ or $\bar{v}$ are given as [13]

$$
\begin{aligned}
& \left(\frac{d \sigma}{d \Omega}\right)_{\frac{v}{\bar{v}}}=\frac{G^{2} \epsilon k}{4 \pi^{2}} \frac{4 \pi}{2 J_{i}+1}\left\{\sum_{J=0}^{\infty}\{(1+\vec{v} \cdot \vec{\beta})\right. \\
& \quad \times\left|\left\langle J_{f}\left\|M_{J}\right\| J_{i}\right\rangle\right|^{2}+[1-\hat{v} \cdot \vec{\beta}+2(\hat{v} \cdot \hat{q})(\hat{q} \cdot \vec{\beta})] \\
& \quad \times\left|\left\langle J_{f}\left\|L_{J}\right\| J_{i}\right\rangle\right|^{2}-\hat{q} \cdot(\hat{v}+\vec{\beta}) 2 \operatorname{Re}\left\langle J_{f}\left\|L_{J}\right\| J_{i}\right\rangle \\
& \left.\quad \times\left\langle J_{f}\left\|M_{J}\right\| J_{i}\right\rangle^{*}\right\}+\sum_{J=1}^{\infty}\{[1-(\hat{v} \cdot \hat{q})(\hat{q} \cdot \vec{\beta})] \\
& \quad \times\left(\left|\left\langle J_{f}\left\|T_{J}^{\mathrm{el}}\right\| J_{i}\right\rangle\right|^{2}+\left|\left\langle J_{f}\left\|T_{J}^{\mathrm{mag}}\right\| J_{i}\right\rangle\right|^{2}\right. \\
& \pm \hat{q} \cdot(\hat{v}-\vec{\beta}) 2 \operatorname{Re}\left[\left\langle J_{f}\left\|T_{J}^{\mathrm{mag}}\right\| J_{i}\right\rangle\right. \\
& \left.\left.\left.\quad \times\left\langle J_{f}\left\|T_{J}^{\mathrm{el}}\right\| J_{i}\right\rangle^{*}\right]\right)\right\}
\end{aligned}
$$

where $\vec{v}$ and $\vec{k}$ are neutrino and lepton momenta, respectively, $\epsilon$ is the lepton energy, $\vec{q}=\vec{k}-\vec{v}, \vec{\beta}=\vec{k} / \epsilon, \hat{v}=\vec{v} /|\vec{v}|$, and $\hat{q}=\vec{q} /|\vec{q}|$.

For charge-exchange reactions, $G=G_{F} \cos \theta_{C}$ with $G_{F}$ the Fermi coupling constant and $\theta_{C}$ the Cabbibo angle, and the lepton is an electron or positron. For neutral current reactions, $G=G_{F}$ and the lepton is a scattered neutrino. The cross section is multiplied by the Fermi function $F\left(Z_{f}, E_{\ell}\right)$ [14], where $Z_{f}$ is the charge of the daughter nucleus and $E_{\ell}$ is the energy of the charged lepton, in the case of charge-exchange reactions.

In Eq. (4), $M_{J}, L_{J}, T_{J}^{\mathrm{el}}$, and $T_{J}^{\mathrm{mag}}$ are Coulomb, longitudinal, transverse electric, and magnetic multipole operators for vector and axial-vector currents defined by

$$
\begin{aligned}
& \left\langle\vec{p}^{\prime}\left|J_{\mu}\right| \vec{p}\right\rangle=i \bar{u}\left(\vec{p}^{\prime}\right)\left[F_{1}^{V} \gamma_{\mu}+F_{2}^{V} \sigma_{\mu \nu} q_{\nu}\right] \tau_{\mp} u(\vec{p}), \\
& \left\langle\vec{p}^{\prime}\left|J_{\mu}^{5}\right| \vec{p}\right\rangle=i \bar{u}\left(\overrightarrow{p^{\prime}}\right)\left[F_{A} \gamma_{5} \gamma_{\mu}-i F_{P} \gamma_{5} q_{\mu}\right] \tau_{\mp} u(\vec{p}),
\end{aligned}
$$

for $\left(v, \ell^{-}\right)$and $\left(\bar{v}, \ell^{+}\right)$reactions. Their matrix elements are given in Ref. [13]. $F_{1}^{V}$ and $F_{2}^{V}$ are isovector electromagnetic form factors, $F_{A}$ is the axial-vector form factor with $F_{A}\left(q_{\mu}^{2}=\right.$ $0)=g_{A}$, and $F_{P}$ is the induced pseudoscalar form factor. Here, we consider vanishing scalar and tensor couplings. In the extreme relativistic limit, when the lepton mass can be neglected, the pseudoscalar coupling in the axial-vector current does not contribute to the neutrino reaction cross sections [13]. The induced pseudoscalar terms, therefore, can be safely neglected in the present calculations, which treat only electrons and positrons, except for neutrinos, because the leptons and neutrino energies are high enough compared to the electron mass.

Equation (4) with the multipole operators obtained for the neutral current,

$$
J_{\mu}^{N}=J_{\mu}^{A_{3}}+J_{\mu}^{V_{3}}-2 \sin ^{2} \theta_{W} J_{\mu}^{\gamma},
$$

TABLE I. Cross sections for the exclusive reaction ${ }^{12} \mathrm{C}\left(v_{e}, e^{-}\right){ }^{12} \mathrm{~N}\left(1_{\mathrm{g} . \mathrm{s}}^{+}\right)$obtained for DAR neutrinos by shell model calculations. The bare $g_{A}$ is used except for the SFO Hamiltonian case, for which $g_{A}^{\text {eff }}=0.95 g_{A}$. For experimental values, the first error is statistical; the second, systematic.

\begin{tabular}{lc}
\hline \hline Hamiltonian & Cross section $\left(\times 10^{-42} \mathrm{~cm}^{2}\right)$ \\
\hline SFO & 9.96 \\
SFO $\left(g_{A}^{\text {eff }}=0.95 g_{A}\right)$ & 9.06 \\
PSDMK2 & 8.48 \\
WBT [4] & 8.42 \\
Hayes-Towner [5] & 8.40 \\
Experiment (LSND [17] $)$ & $8.9 \pm 0.3 \pm 0.9$ \\
Experiment & $9.1 \pm 0.5 \pm 0.8$ \\
$\quad$ KARMEN [18] $)$ & \\
\hline \hline
\end{tabular}

with

$$
\begin{aligned}
J_{\mu}^{\gamma} & =J_{\mu}^{S}+J_{\mu}^{V_{3}}, \\
J_{\mu}^{A_{3}} & =i \vec{u}\left(\vec{p}^{\prime}\right)\left[F_{A} \gamma_{5} \gamma_{\mu}-i F_{P} \gamma_{5} q_{\mu}\right] \frac{\tau_{3}}{2} u(\vec{p}), \\
J_{\mu}^{V_{3}} & =i \vec{u}\left(\vec{p}^{\prime}\right)\left[F_{1}^{V} \gamma_{\mu}+F_{2}^{V} \sigma_{\mu \nu} q_{\nu}\right] \frac{\tau_{3}}{2} u(\vec{p}), \\
J_{\mu}^{S} & =i \bar{u}\left(\vec{p}^{\prime}\right)\left[F_{1}^{S} \gamma_{\mu}+F_{2}^{S} \sigma_{\mu \nu} q_{\nu}\right] \frac{1}{2} u(\vec{p}),
\end{aligned}
$$

applies also to $\left(v, v^{\prime}\right)$ and $\left(\bar{v}, \bar{v}^{\prime}\right)$ reactions. Here, $\theta_{W}$ is the Weinberg angle, and $F_{1}^{S}$ and $F_{2}^{S}$ are isoscalar electromagnetic form factors. As mentioned above, the contributions from the pseudoscalar coupling $F_{P}$ vanish.

First, we show results of cross sections for the exclusive reaction ${ }^{12} \mathrm{C}\left(v_{e}, e^{-}\right){ }^{12} \mathrm{~N}\left(1_{\text {g.s. }}^{+}\right)$induced by DAR neutrinos. Calculated cross sections obtained by using the SFO and the (PSDMK2) $[1,15,16]$ shell model Hamiltonians within the configuration space including up to $2 \hbar \omega$ excitations are given in Table I as well as the observed values [17,18]. Harmonic oscillator wave functions with a size parameter $b=1.64 \mathrm{fm}$ are used. The axial electric dipole $\left(E_{5} 1\right)$ and the magnetic dipole $(M 1)$ terms contribute to the GamowTeller transition. There are also contributions from the axial Coulomb and longitudinal dipole $\left(C_{5} 1\right.$ and $\left.L_{5} 1\right)$ terms, but they are rather small. The bare axial vector coupling constant, $g_{A}=-1.263$ and an effective one with $g_{A}^{\text {eff }}=0.95 g_{A}$ are used. The latter reproduces the experimental $B(G T)$ value for the transition to ${ }^{12} \mathrm{~N}\left(1_{\text {g.s. }}^{+}\right)$. While the SFO Hamiltonian gives larger values of the cross section than those obtained by other conventional shell model Hamiltonians [4,5], the calculated cross sections are found to be consistent with the experimental ones within the experimental errors [17,18]. A no core shell model $(\mathrm{NCSM})$ calculation gives a smaller value of $6.80 \times$ $10^{-42} \mathrm{~cm}^{2}$ for the cross section [19], while a continuum random phase approximation (CRPA) method gives a larger value of $13.88 \times 10^{-42} \mathrm{~cm}^{2}$ [20].

Next, we show in Table II calculated results of the cross sections for exclusive neutral current reactions on ${ }^{12} \mathrm{C}$, that is, $\left(v_{e}, v_{e}^{\prime}\right),\left(\bar{v}_{\mu}, \bar{v}_{\mu}^{\prime}\right)$, and $\left(v_{\mu}, v_{\mu}^{\prime}\right)$ reactions leading to the $1^{+}$ ( $T=1,15.1 \mathrm{MeV}$ ) state of ${ }^{12} \mathrm{C}$ induced by the DAR neutrinos. 
TABLE II. Cross sections for exclusive neutral current reaction on ${ }^{12} \mathrm{C}$ leading to the $1^{+}(T=1,15.1 \mathrm{MeV})$ state induced by DAR neutrinos; $v_{e}$ and $\bar{v}_{\mu}$ from $\mu^{+}$decay as well as $v_{\mu}$ from $\pi^{+}$decay. Experimental values of the sum of $v_{e}$ - and $\bar{v}_{\mu}$-induced reaction cross sections [18] and that of $v_{\mu}$-induced reaction cross section [21] are included.

\begin{tabular}{|c|c|c|c|c|}
\hline \multirow[t]{2}{*}{ Hamiltonian } & \multicolumn{4}{|c|}{ Cross sections $\left(\times 10^{-42} \mathrm{~cm}^{2}\right)$} \\
\hline & $\left(v_{e}, v_{e}^{\prime}\right)$ & $\left(\bar{v}_{\mu}, \bar{v}_{\mu}^{\prime}\right.$ & $\left(v_{e}, v_{e}^{\prime}\right)+\left(\bar{v}_{\mu}, \bar{v}_{\mu}^{\prime}\right)$ & $\left(v_{\mu}, v_{\mu}^{\prime}\right)$ \\
\hline $\begin{array}{c}\mathrm{SFO}\left(g_{A}^{\mathrm{eff}}=\right. \\
\left.0.95 g_{A}\right)\end{array}$ & 4.44 & 5.32 & 9.76 & 2.68 \\
\hline PSDMK2 & 3.75 & 4.52 & 8.27 & 2.26 \\
\hline $\begin{array}{l}\text { Experiment } \\
\text { (KARMEN) }\end{array}$ & & & $\begin{array}{c}10.4 \pm 1.0 \pm 0.9 \\
{[18]}\end{array}$ & $\begin{array}{c}3.2 \pm 0.5 \pm 0.4 \\
{[21]}\end{array}$ \\
\hline
\end{tabular}

Experimental value of the cross section for the sum of the $\left(v_{e}, v_{e}^{\prime}\right)$ and $\left(\bar{v}_{\mu}, \bar{v}_{\mu}^{\prime}\right)$ reactions is available [18] (see Table II). The calculated cross section obtained for the SFO Hamiltonian is found to be close to the observed value, while that for the PSDMK2 Hamiltonian is smaller than the experimental one about by $20 \%$. Note that the $\mathrm{B}(\mathrm{GT})$ value obtained for the PSDMK2 Hamiltonian with the $(0-2) \hbar \omega$ configuration space is smaller than the observed one by $16 \%$ [1]. Experimental value of the $\left(v_{\mu}, v_{\mu}^{\prime}\right)$ reaction cross section [21] is also found to be consistent with the calculated value for the SFO Hamiltonian, whereas it is a bit larger than the calculated value for the PSDMK2 Hamiltonian.

Finally, we show in Table III calulated cross sections for the ${ }^{12} \mathrm{C}\left(v_{e}, e^{-}\right){ }^{12} \mathrm{~N}^{*}$ reaction leading to excited states of ${ }^{12} \mathrm{~N}$ obtained by the shell models with the configuration space including up to $3 \hbar \omega$ excitations. The multipolarities up to $J=3$ are included here. The contributions from the spindipole transitions to the $0^{-}, 1^{-}$, and $2^{-}$states are dominant. There are also some contributions from other multipolarities, $2^{+}, 3^{-}, 3^{+}$, and $0^{+}$, as well as $1^{+}$except for the ground state. The SFO Hamiltonian gives closer energy levels for the negative parity states than does the PSDMK2 Hamiltonian. The excitation energies of the first $0^{-}, 1^{-}, 2^{-}$, and $3^{-}$states with $T=1$ are 20.304 (21.086), 19.053 (20.137), 17.823 (18.881), and 19.087 (20.128) MeV, respectively, for the SFO (PSDMK2) case, while experimental values are $17.230\left(1^{-}\right)$,

TABLE III. Cross sections for the reaction process ${ }^{12} \mathrm{C}$ $\left(v_{e}, e^{-}\right){ }^{12} \mathrm{~N}^{*}$ obtained for DAR neutrinos by shell model calculations. Bare $g_{A}$ is used unless specified.

\begin{tabular}{lc}
\hline \hline Hamiltonian & Cross section $\left(\times 10^{-42} \mathrm{~cm}^{2}\right)$ \\
\hline SFO & 8.35 \\
SFO $\left(g_{A}^{\text {eff }}=0.70 g_{A}\right)$ & 5.22 \\
PSDMK2 & 7.14 \\
PSDMK2 $\left(g_{A}^{\text {eff }}=0.75 g_{A}\right)$ & 4.87 \\
WBT [4] & 8.31 \\
Hayes-Towner [5] & 3.80 \\
Experiment $(L S N D ~[17])$ & $4.3 \pm 0.4 \pm 0.6$ \\
Experiment & $5.1 \pm 0.6 \pm 0.5$ \\
$\quad$ KARMEN [22] $)$ & \\
\hline \hline
\end{tabular}
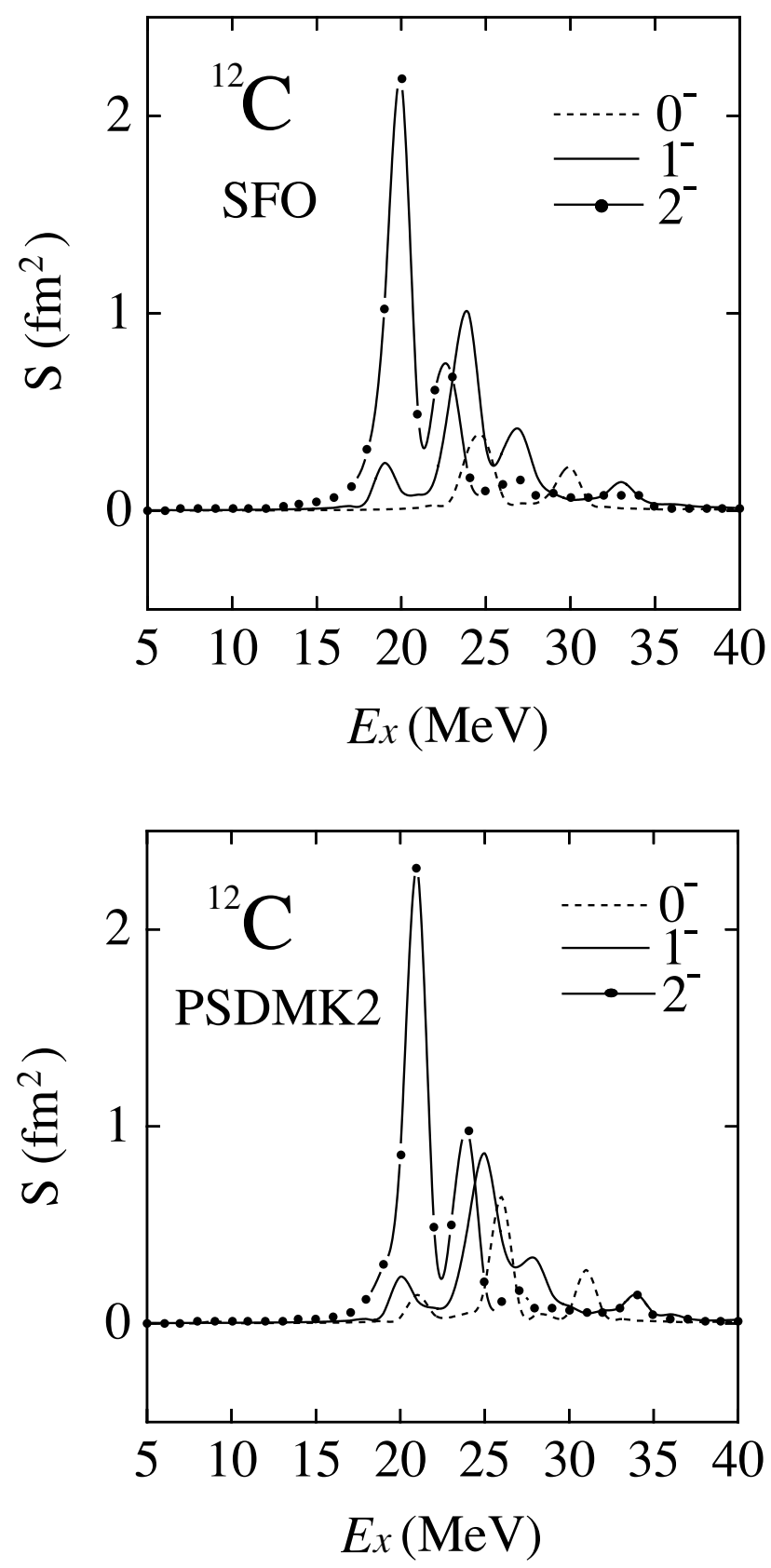

FIG. 3. Spin-dipole strengths in ${ }^{12} \mathrm{C}$ for the SFO and PSDMK2 Hamiltonians. Strengths are folded by a Lorenzian with the width of $1 \mathrm{MeV}$.

$16.570\left(2^{-}\right)$, and $18.350 \mathrm{MeV}\left(3^{-}\right)$. The spin-dipole strengths obtained by the SFO and PSDMK2 Hamiltonians are shown in Fig. 3. Calculated strengths summed up to the excitation energy of $E_{x}=50 \mathrm{MeV}$ are 1.79 (1.78), 4.33 (4.12), and 7.19 (7.03) $\mathrm{fm}^{2}$ for the $0^{-}, 1^{-}$, and $2^{-}$states, respectively, for the SFO (PSDMK2) Hamiltonian. The centroid energies defined by the energy-weighted sum divided by the non-energyweighted sum of the strength are calculated to be 25.9 (26.8), 25.3 (26.2), and 21.5 (22.7) MeV for the SFO (PSDMK2) case for the $0^{-}, 1^{-}$, and $2^{-}$states, respectively. The strength by the SFO Hamiltonian is shifted toward the lower energy region by 
about $1 \mathrm{MeV}$ compared to the PSDMK2 Hamiltonian, while the total strength is increased by only about $3 \%$.

Shell model calculations give larger cross sections than the observed values $[17,22]$ except for the one by Hayes and Towner [5], who used Woods-Saxon wave functions instead of harmonic oscillator wave functions. A CRPA calculation [20] gives a cross section close to the experiment. Effective axialvector coupling constants with quenching factors, $g_{A}^{\text {eff }} / g_{A}=$ 0.7 and 0.75 , are adopted for the SFO and PSDMK2 Hamiltonians, respectively. Shell model calculations with these quenching factors reproduce the experimental cross section.

Observation of electron scattering and $(p, n)$ reaction data indicates that the spin-dipole strength in the $2^{-}(T=1)$ state in ${ }^{12} \mathrm{C}\left({ }^{12} \mathrm{~N}\right)$ at $E_{x}=19.40(4.14) \mathrm{MeV}$ is considerably quenched by a factor of about 2 [23-25]. Note that the $2^{-}$state exhausts about $60 \%$ of the total spin-dipole strength for $2^{-}$states (see also Ref. [25]). This results in greater importance for the $2^{-}$ state in the cross section; that is, about $75 \%$ of the cross section for ${ }^{12} \mathrm{C}\left(v_{e}, e^{-}\right){ }^{12} \mathrm{~N}^{*}\left(2^{-}\right)$induced by DAR neutrinos comes from the $2^{-}$state at $4.14 \mathrm{MeV}$ because of the neutrino energy cut-off at $52.8 \mathrm{MeV}$. The $M 2$ form factor for ${ }^{12} \mathrm{C}\left(e, e^{\prime}\right)$ ${ }^{12} \mathrm{C}\left(2^{-}, T=1,19.40 \mathrm{MeV}\right)$ was obtained in the momentum transfer region of $q=0.3 \sim 1.0 \mathrm{fm}^{-1}$ [23]. The observed form factor is found to be consistent with a large quenching of the spin $g$ factor: $g_{s}^{\text {eff }} / g_{s}=0.70 \pm 0.05(0.75 \pm 0.05)$ for the SFO (PSDMK2) Hamiltonian. This was also pointed out in Ref. [25], where $g_{s}^{\text {eff }} / g_{s}=0.65$ was obtained for the Cohen-Kurath Hamiltonian. The $(p, n)$ and $\left(d,{ }^{2} \mathrm{He}\right)$ reaction data support similar order of large quenching factors [24-26]. More experimental investigation is important and necessary to acquire systematic information on the nature of quenching of the spin-dipole strength. Quenching due to the coupling to many-particle many-hole states at high excitation energies could be larger for the spin-dipole states that lie above the Gamow-Teller state because of a smaller energy-difference denominator.

In the case of the $2^{+}(T=1)$ state, $(p, n)$ and $\left(p, p^{\prime}\right)$ reaction data indicate that the transition strength to the $2^{+}(T=1)$ state in ${ }^{12} \mathrm{~N}\left({ }^{12} \mathrm{C}\right)$ at $E_{x}=0.96(16.11) \mathrm{MeV}$ is quenched by a factor of about 2 [27,28]. It was also found in Ref. [29] that in the electric dipole transitions in ${ }^{12} \mathrm{C}$, the reduction of the calculated cross section by a multiplying factor of 0.7 was necessary to obtain quantitative agreement with the available experimental cross section [30]. This suggests the importance of the coupling to many-particle many-hole states with excitations larger than $3 \hbar \omega$. These observations support the necessity for the large quenching of $g_{A}$ in the inclusive reactions.

\section{B. Reactions on ${ }^{12} \mathrm{C}$ induced by supernova neutrinos}

We study charge-exchange and neutral current reactions on ${ }^{12} \mathrm{C}$ induced by the supernova neutrinos. Fermi distribution functions are employed for the spectra of supernova neutrinos. The value of the chemical potential is set to be zero. Average energies of supernova neutrinos are about 10, 15, and 15 25 MeV for $v_{e}, \bar{v}_{e}$, and $v_{\mu, \tau}$, respectively [31]. The neutrino temperature of the Fermi distribution is about onethird of the average energy.
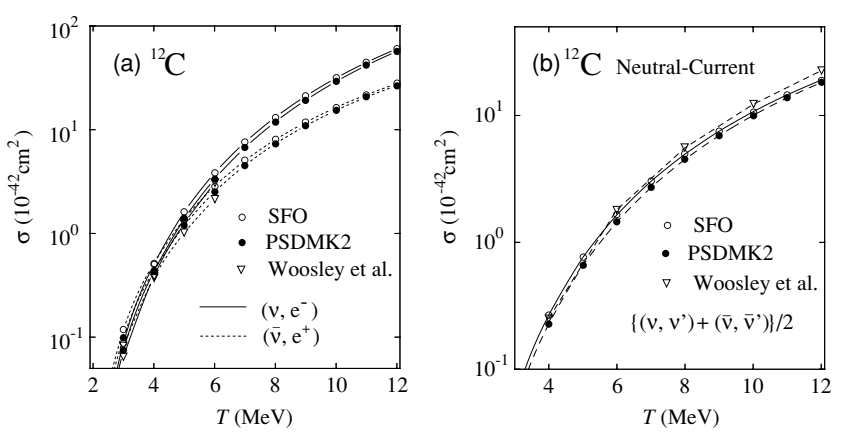

FIG. 4. Calculated cross sections for neutrino ${ }^{12} \mathrm{C}$ reactions induced by supernova neutrinos with temperature $T$ obtained by using the SFO and PSDMK2 Hamiltonians. Both (a) the charge-exchange $\left(v_{e}, e^{-}\right)$and $\left(\bar{v}_{e}, e^{+}\right)$reactions and (b) the neutral current reactions are treated. Average values of the $\left(v, v^{\prime}\right)$ and $\left(\bar{v}, \bar{v}^{\prime}\right)$ cross sections are shown for the neutral current reactions. Previous calculations of Ref. [6] are also given.

Calculated cross sections for supernova neutrinos with temperature $T=2 \sim 12 \mathrm{MeV}$ are shown in Fig. 4 for the SFO and PSDMK2 Hamiltonians. The axial-vector coupling constants which reproduce the experimental $\left(v_{e}, e^{-}\right)$cross section for the DAR neutrinos are adopted. The values of $g_{A}^{\text {eff }} / g_{A}$ are 0.95 and 0.70 for the exclusive reaction and the transitions to the excited states, respectively, in the case of the SFO Hamiltonian. Those employed for the PSDMK2 Hamiltonian are 1.0 for the exclusive reaction and 0.75 for the transitions to the excited states. We will use these values for $g_{A}^{\text {eff }}$ hereafter. As for the neutral current reactions, contributions from the isoscalar transitions are not included in the calculations as they are quite small. Calculated cross sections for the SFO are enhanced compared with those for the PSDMK2 in both charge-exchange and neutral current reactions. The charge-exchange reaction cross sections are also enhanced compared with the previous calculations by Woosley et al. [6], in which the configurations are restricted to up to $1 \hbar \omega$ excitations with $g_{A}^{\text {eff }} / g_{A}=0.7$ for the excitations of negative-parity states.

Branching ratios from each excited level are calculated for decay channels involving neutron, proton, $\alpha$, and $\gamma$ by the Hauser-Feshbach statistical model [32]. All the levels obtained by the present shell model calculations are adopted as levels in the decaying and daughter nuclei with specific isospin assignments.

The particle transmission coefficients are calculated by the optical model with conventional potentials $[33,34]$ at selected grid energies, and they are interpolated by using a spline interpolation. Weights proportional to the square of the isospin Clebsch-Gordan coefficients are multiplied to the transmission coefficients obtained by the optical model to account for the isospin conservation. We ignored any isospin mixing, which may be significant for some of the light nuclei. The $\gamma$-transmission coefficients are calculated with a simple Brink's formula. The $E 1$ and $M 1$ parameters were taken from the RIPL-2 database [35]. The $\gamma$ cascade in the initial excited nuclei and subsequent decay were fully considered. 

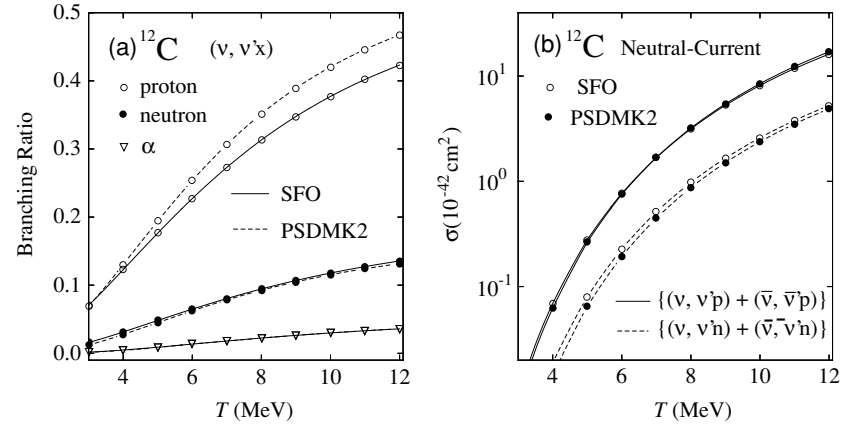

FIG. 5. (a) Branching ratios for proton, neutron, and $\alpha$ emission channels for neutral current reactions on ${ }^{12} \mathrm{C}$ obtained by the Hauser-Feshbach theory. (b) Calculated cross sections for proton and neutron knock-out channels obtained by using the SFO and PSDMK2 Hamiltonians.

Calculated branching ratios as well as the proton and neutron emission cross sections are shown in Fig. 5 for the neutral current reactions. The branching ratios for the proton and neutron emissions obtained by the PSDMK2 Hamiltonian are close to those of Ref. [6]. The branching ratios for the proton emissions depend on the Hamiltonians, SFO or PSDMK2, while the neutron emission cross sections are found to be enhanced for the SFO case.

Neutral current reactions, ${ }^{12} \mathrm{C}\left(\nu, v^{\prime} p\right){ }^{11} \mathrm{~B}$ and ${ }^{12} \mathrm{C}\left(\nu, v^{\prime} n\right)$ ${ }^{11} \mathrm{C}\left(\beta^{+}\right){ }^{11} \mathrm{~B}$, are important for the production of ${ }^{11} \mathrm{~B}$ in the $\mathrm{He}-\mathrm{C}$ layer and O-rich layer during supernova explosions. The effects of the reactions on the abundance of ${ }^{11} \mathrm{~B}$ in the supernovae will be discussed in Sec. IV.

\section{Reactions on ${ }^{4} \mathrm{He}$}

We treat here the ${ }^{4} \mathrm{He}$ nucleus for the study of neutrinonucleus reactions. The reaction cross section on the nucleus has an important role in determining abundances of light elements such as ${ }^{7} \mathrm{Li}$ and ${ }^{11} \mathrm{~B}$ during supernova explosions.

The $\nu_{-}{ }^{4} \mathrm{He}$ reactions are induced dominantly by excitations of spin-dipole states. The Warburton-Brown (WBP) [36] and Millener-Kurath (SPSDMK) [15,16] Hamiltonians are used for the shell model calculations of ${ }^{4} \mathrm{He}$ with configurations including up to $3 \hbar \omega$ excitations. In the SPSDMK interaction, the Cohen-Kurath interaction, (8-16)POT [7], is used for the $p$-shell part, while the Millener-Kurath interaction is used for the cross-shell matrix elements between $0 s$ and $0 p$ as well as $0 p$ and $1 s 0 d$ orbits. The $s d$-shell part is the Preedom-Wildenthal interaction [37], and all others are Kuo's renormalized $G$ matrices [38].

Calculated spin-dipole strengths are shown in Fig. 6. Harmonic oscillator wave functions with a size parameter $b=1.38 \mathrm{fm}$ are used. The strength is more fragmented in the case of the WBP Hamiltonian. The summed strengths are $3.34 \mathrm{fm}^{2}$ for the WBP and $4.71 \mathrm{fm}^{2}$ for the SPSDMK Hamiltonians, up to the excitation energy of $E_{x}=50 \mathrm{MeV}$.

Calculated cross sections for the charge-exchange and neutral current reactions are shown in Fig. 7 for the supernova neutrinos with $T=2 \sim 12 \mathrm{MeV}$. The bare $g_{A}$ is employed. The cross sections for the SPSDMK case are found to be larger than
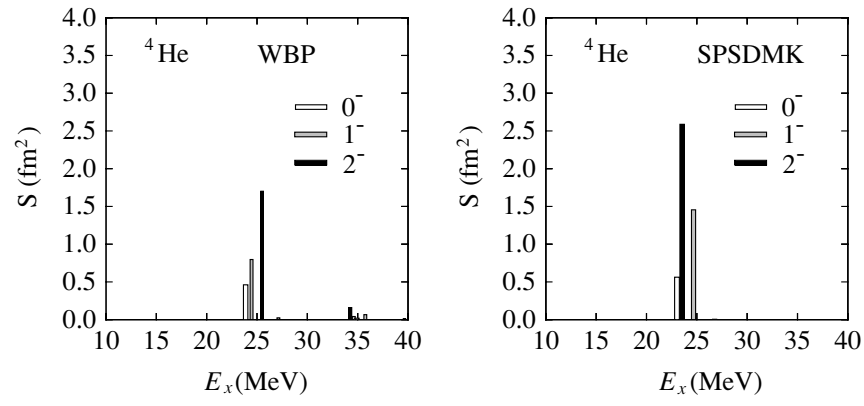

FIG. 6. Spin-dipole strengths in ${ }^{4} \mathrm{He}$ for the WBP and SPSDMK Hamiltonians.

those for the WBP case. They are both enhanced compared with the previous calculations [6], which used Sussex matrix elements [9] for the effective interaction with a larger harmonic oscillator size parameter of $b=1.5 \mathrm{fm}$.

A recent microscopic ab initio calculation of the neutral current reaction on ${ }^{4} \mathrm{He}$ with a realistic nucleon-nucleon interaction, $A V 8^{\prime}$ [39], predicts cross sections with a steeper dependence on the neutrino temperature than the shell model calculations [40]. At $T=10 \mathrm{MeV}$, the calculated cross section in Ref. [40] is close to that obtained by the WBP Hamiltonian, while at $T=12 \mathrm{MeV}$ it is enhanced by about $16 \%$. At $T=$ $8 \mathrm{MeV}$, on the other hand, the WBP Hamiltonian predicts a larger cross section by about $15 \%$.

The neutral current reactions, ${ }^{4} \mathrm{He}\left(v, v^{\prime} p\right){ }^{3} \mathrm{H}$ and ${ }^{4} \mathrm{He}$ $\left(v, v^{\prime} p\right){ }^{3} \mathrm{He}$, are important for the production of ${ }^{7} \mathrm{Li}$ through ${ }^{3} \mathrm{H}(\alpha, \gamma){ }^{7} \mathrm{Li}$ and ${ }^{3} \mathrm{He}(\alpha, \gamma){ }^{7} \mathrm{Be}\left(e^{-}, v_{e}\right){ }^{7} \mathrm{Li}$ processes in the He-C layer during supernova explosions. The reactions are also important for the production of ${ }^{11} \mathrm{~B}$, as the abundance of ${ }^{7} \mathrm{Li}$ affects the production of ${ }^{11} \mathrm{~B}$ through the process ${ }^{7} \mathrm{Li}(\alpha, \gamma)$ ${ }^{11} \mathrm{~B}$.

\section{ABUNDANCES OF ${ }^{7} \mathrm{Li}$ AND ${ }^{11}$ B DURING SUPERNOVA EXPLOSIONS}

The enhancement of $\nu_{-}{ }^{4} \mathrm{He}$ and $\nu_{-12} \mathrm{C}$ reaction cross sections affects the abundances of ${ }^{7} \mathrm{Li}$ and ${ }^{11} \mathrm{~B}$ in the nucleosynthesis process during supernova explosions. The nucleosynthesis path of light elements is shown in Fig. 8. The neutral current reactions, ${ }^{12} \mathrm{C}\left(v, v^{\prime} p\right){ }^{11} \mathrm{~B}$ and ${ }^{12} \mathrm{C}\left(v, v^{\prime} n\right)$
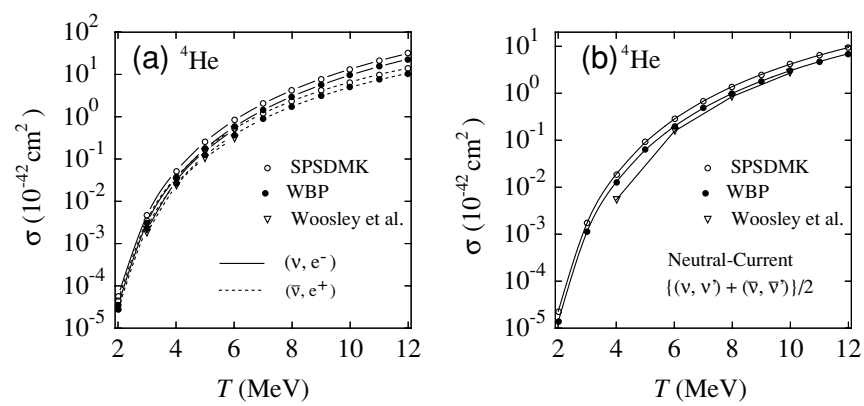

FIG. 7. Calculated (a) charge-exchange and (b) neutral current reaction cross sections for $v_{-}^{4} \mathrm{He}$ reactions obtained by using the WBP and SPSDMK Hamiltonians. Previous calculations of Ref. [6] are also shown. 
TABLE IV. Production yields of ${ }^{7} \mathrm{Li}$ and ${ }^{11} \mathrm{~B}$ in a supernova explosion model.

\begin{tabular}{lcc}
\hline \hline Hamiltonians & $M\left({ }^{7} \mathrm{Li}\right) / M_{\odot}$ & $M\left({ }^{11} \mathrm{~B}\right) / M_{\odot}$ \\
\hline WBP+SFO & $3.06 \times 10^{-7}$ & $7.51 \times 10^{-7}$ \\
SPSDMK + & $4.24 \times 10^{-7}$ & $9.38 \times 10^{-7}$ \\
$\quad$ PSDMK2 & & \\
HW92 & $2.36 \times 10^{-7}$ & $6.29 \times 10^{-7}$ \\
\hline \hline
\end{tabular}

${ }^{11} \mathrm{C}$, are important for the production of ${ }^{11} \mathrm{~B}$. If these cross sections are enhanced, the abundance of ${ }^{11} \mathrm{~B}$ is increased. The reactions, ${ }^{4} \mathrm{He}\left(v, v^{\prime} p\right){ }^{3} \mathrm{H}$ and ${ }^{4} \mathrm{He}\left(\nu, v^{\prime} n\right){ }^{3} \mathrm{He}$ are important for the production of ${ }^{7} \mathrm{Li}$ through ${ }^{3} \mathrm{H}(\alpha, \gamma){ }^{7} \mathrm{Li}$ and ${ }^{3} \mathrm{He}$ $(\alpha, \gamma){ }^{7} \mathrm{Be}\left(e^{-}, v_{e}\right){ }^{7} \mathrm{Li}$ processes. If the $\nu_{-}{ }^{4} \mathrm{He}$ reaction cross sections are enhanced, the abundances of both ${ }^{7} \mathrm{Li}$ and ${ }^{11} \mathrm{~B}$ are increased, because the abundance of ${ }^{11} \mathrm{~B}$ is affected by that of ${ }^{7} \mathrm{Li}$ through ${ }^{7} \mathrm{Li}(\alpha, \gamma){ }^{11} \mathrm{~B}$, etc.

To investigate the effects of our new reaction cross sections for $v-{ }^{4} \mathrm{He}$ and $\nu-{ }^{12} \mathrm{C}$ on the yields of ${ }^{7} \mathrm{Li}$ and ${ }^{11} \mathrm{~B}$ in a core-collapse supernova, we carry out a detailed calculation of nucleosynthesis during supernova explosions. The supernova explosion model is the same as in $[41,42]$. The progenitor structure is adopted from a $16.2 M_{\odot}$ presupernova model corresponding to SN 1987A [43]. The nuclear reaction network consists of 291 species of nuclei. The luminosity and energy spectra of neutrinos are important for neutrino-nucleus interactions. We assume that the neutrino luminosity decays exponentially in time with a time scale of $3 \mathrm{~s}$ and is equally partitioned among three flavors of neutrinos and antineutrinos. The neutrino energy spectra are assumed to obey Fermi distributions with zero-chemical potentials. We set the total neutrino energy to be $3 \times 10^{53}$ erg and the neutrino temperatures of $v_{e}, \bar{v}_{e}$, and $v_{\mu, \tau}$ and $\bar{v}_{\mu, \tau}$ to be $T_{v_{e}}=3.2$, $T_{\bar{v}_{e}}=5.0$, and $T_{v_{\mu, \tau}}=6.0 \mathrm{MeV}$ respectively [41,42], as the standard case.

We show in Table IV production yields of ${ }^{7} \mathrm{Li}$ and ${ }^{11} \mathrm{~B}$ in the nucleosynthesis during the supernova explosion obtained by using the cross sections of the two sets of the shell model Hamiltonians; one by WBP for ${ }^{4} \mathrm{He}$ and SFO for ${ }^{12} \mathrm{C}$ and the other by SPSDMK for ${ }^{4} \mathrm{He}$ and PSDMK2 for ${ }^{12} \mathrm{C}$. The production yields obtained by using the cross sections of Hoffman and Woosley (HW92) [44] are also given. Compared to the case for HW92, the abundances of ${ }^{7} \mathrm{Li}$ and ${ }^{11} \mathrm{~B}$ are

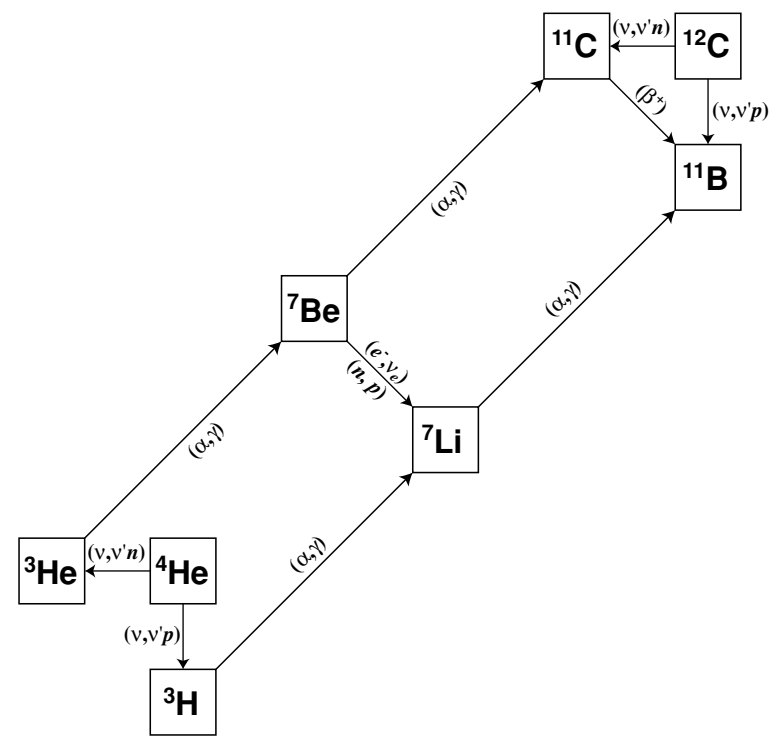

FIG. 8. Nucleosynthesis path of light elements ${ }^{7} \mathrm{Li}$ and ${ }^{11} \mathrm{~B}$ during supernova explosions.

enhanced by a factor of 1.30 and 1.19 , respectively, for $\mathrm{WBP}+\mathrm{SFO}$, while they are enhanced more, by a factor of 1.79 and 1.49, respectively, for SPSDMK+PSDMK2. Enhancement factors for ${ }^{7} \mathrm{Li}$ are larger than those for ${ }^{11} \mathrm{~B}$, because the cross sections for ${ }^{4} \mathrm{He}$ are more enhanced than those for ${ }^{12} \mathrm{C}$. We find that about $40 \%$ of the production of ${ }^{11} \mathrm{~B}$ is caused by the $\nu-{ }^{12} \mathrm{C}$ reactions, while the other $60 \%$ is due to the $\nu-{ }^{4} \mathrm{He}$ reactions.

We investigate the production yields of ${ }^{7} \mathrm{Li}$ and ${ }^{11} \mathrm{~B}$ by changing the temperature of $v_{e}, \bar{v}_{e}$, and $v_{\mu, \tau}$. We set the following restrictions to the temperature: (1) $T_{\nu_{e}}<T_{\bar{\nu}_{e}}<$ $T_{v_{\mu, \tau}}$, (2) $T_{\bar{v}_{e}} \leqslant 5 \mathrm{MeV}$, and (3) $T_{v_{\mu, \tau}} / T_{\bar{\nu}_{e}} \simeq 1.2$. We further choose temperature so that the production yield of ${ }^{11} \mathrm{~B}$ ranges between $3.3 \times 10^{-7} M_{\odot}$ and $7.4 \times 10^{-7} M_{\odot}$ in order to satisfy the supernova contribution of $\mathrm{B}$ abundance in the galactic chemical evolution $[42,45]$. The total neutrino energy is varied within the estimated error bar for the released gravitational binding energy of a proto-neutron star [46]. Calculated results are given in Table V. Although the production yield of ${ }^{11} \mathrm{~B}$ depends little on the Hamiltonians, the production yield of ${ }^{7} \mathrm{Li}$ is slightly larger for the case of SPSDMK+PSDMK2 than for the case of $\mathrm{WBP}+\mathrm{SFO}$.

TABLE V. Dependence of the production yields of ${ }^{7} \mathrm{Li}$ and ${ }^{11} \mathrm{~B}$ on neutrino temperatures and total neutrino energy. Reaction cross sections by (a) WBP and SFO Hamiltonians and (b) SPSDMK and PSDMK2 Hamiltonians for ${ }^{4} \mathrm{He}$ and ${ }^{12} \mathrm{C}$, respectively, are used.

\begin{tabular}{|c|c|c|c|c|c|c|c|}
\hline Hamiltonians & Neutrino model & $\begin{array}{c}T_{v_{e}} \\
(\mathrm{MeV})\end{array}$ & $\begin{array}{c}T_{\bar{v}_{e}} \\
(\mathrm{MeV})\end{array}$ & $\begin{array}{c}T_{v_{\mu, \tau}} \\
(\mathrm{MeV})\end{array}$ & $\begin{array}{c}E_{v} \\
\left(\times 10^{53} \text { erg }\right)\end{array}$ & $\begin{array}{c}M\left({ }^{7} \mathrm{Li}\right) \\
\left(M_{\odot}\right)\end{array}$ & $\begin{array}{c}M\left({ }^{11} \mathrm{~B}\right) \\
\left(M_{\odot}\right)\end{array}$ \\
\hline \multirow[t]{3}{*}{ (a) $\mathrm{WBP}+\mathrm{SFO}$} & Low $T_{v}$, high $E_{v}$ & 3.2 & 4.1 & 5.0 & 3.53 & $1.51 \times 10^{-7}$ & $3.59 \times 10^{-7}$ \\
\hline & $\operatorname{Med} T_{v}$, med $E_{v}$ & 3.2 & 4.8 & 5.8 & 3.0 & $2.62 \times 10^{-7}$ & $6.36 \times 10^{-7}$ \\
\hline & High $T_{v}$, low $E_{v}$ & 3.2 & 5.0 & 6.4 & 2.35 & $3.13 \times 10^{-7}$ & $7.45 \times 10^{-7}$ \\
\hline \multirow[t]{3}{*}{ (b) SPSDMK+PSDMK2 } & Low $T_{v}$, high $E_{v}$ & 3.2 & 4.0 & 4.8 & 3.53 & $1.76 \times 10^{-7}$ & $3.55 \times 10^{-7}$ \\
\hline & $\operatorname{Med} T_{v}$, med $E_{v}$ & 3.2 & 4.6 & 5.6 & 3.0 & $3.07 \times 10^{-7}$ & $6.57 \times 10^{-7}$ \\
\hline & High $T_{v}$, low $E_{v}$ & 3.2 & 5.0 & 6.0 & 2.35 & $3.41 \times 10^{-7}$ & $7.35 \times 10^{-7}$ \\
\hline
\end{tabular}


When we take into account the effect of neutrino oscillations, charge-exchange reactions have an additional role in increasing both ${ }^{7} \mathrm{Li}$ and ${ }^{11} \mathrm{~B}$ yields. The production yields prove to be sensitive to the mixing angles, in particular to $\theta_{13}$, and mass hierarchy [41]. This subject coupled with the use of our new reaction cross sections, will be discussed in Ref. [47].

\section{SUMMARY}

Neutrino-nucleus reactions on ${ }^{12} \mathrm{C}$ induced by DAR neutrinos and supernova neutrinos are investigated by using a new shell model Hamiltonian for $p$-shell nuclei, called SFO, which takes into accout important roles of spin-isospin interactions.

First, the monopole terms of the tensor components of the SFO interaction are shown to have proper signs, that is, the $p-n$ interaction is attractive between $j_{\rangle}$and $j_{\langle}$orbits but repulsive between $j_{\rangle}$and $j_{\rangle}$or $j_{\langle}$and $j_{\langle}$orbits. This zigzag structure of the tensor interaction is pointed out to be important in realizing the proper evolution of effective single-particle energies toward the drip lines. For $N=8$ isotones, the shell gap between the $0 p_{1 / 2}$ and $0 p_{3 / 2}$ orbits is shown to increase near the neutronrich side.

Cross sections of charge-exchange exclusive and inclusive reactions on ${ }^{12} \mathrm{C}$ are then obtained for the DAR neutrinos with the use of the SFO Hamiltonian and compared with experimental values. The exclusive reaction is found to be well reproduced with $g_{A}^{\text {eff }}=0.95 g_{A}$. A quenching of $g_{A}$
$\left(g_{A}^{\text {eff }}=0.7 g_{A}\right)$ is found to be necessary to explain the cross section for excited states.

Charge-exchange and neutral current reactions are studied also for supernova neutrinos. Branching ratios to proton, neutron, $\alpha$, and $\gamma$ emission channels are calculated by the Hauser-Feshbach theory, and cross sections for $\left(v, v^{\prime} p\right)$ and $\left(\nu, v^{\prime} n\right)$ reactions are obtained. Calculated cross sections are found to be enhanced compared with those by the PSDMK2 Hamiltonian.

Neutrino- ${ }^{4} \mathrm{He}$ reactions are also investigated by using the WBP and the SPSDMK Hamiltonians. Calculated cross sections are enhanced compared with previous calculations of Ref. [6]. A possible consequence of the enhancement of the $v_{-}{ }^{4} \mathrm{He}$ and $\nu-{ }^{12} \mathrm{C}$ reaction cross sections on the abundances of light elements is discussed. The production yields of ${ }^{7} \mathrm{Li}$ and ${ }^{11} \mathrm{~B}$ are found to be enhanced during supernova explosions.

\section{ACKNOWLEDGMENTS}

The authors would like to thank Professor A. Gelberg for the careful reading of the manuscript. This work has been supported in part by Grants-in-Aid for Scientific Research $(14540271,17540275,18540290)$ and for Specially Promoted Research (13002001) of the Ministry of Education, Culture, Sports, Science and Technology of Japan, and the Mitsubishi Foundation.
[1] T. Suzuki, R. Fujimoto, and T. Otsuka, Phys. Rev. C 67, 044302 (2003).

[2] T. Otsuka, R. Fujimoto, Y. Utsuno, B. A. Brown, M. Honma, and T. Mizusaki, Phys. Rev. Lett. 87, 082502 (2001).

[3] T. Otsuka, T. Suzuki, R. Fujimoto, H. Grawe, and Y. Akaishi, Phys. Rev. Lett. 95, 232502 (2005).

[4] C. Volpe, N. Auerbach, G. Colò, T. Suzuki, and N. Van Giai, Phys. Rev. C 62, 015501 (2000).

[5] A. C. Hayes and I. S. Towner, Phys. Rev. C 61, 044603 (2000).

[6] S. E. Woosley, D. H. Hartmann, R. D. Hoffman, and W. C. Haxton, Astrophys. J. 356, 272 (1990).

[7] S. Cohen and D. Kurath, Nucl. Phys. 73, 1 (1965).

[8] M. W. Kirson, Phys. Lett. B47, 110 (1973); I. Kakkar and Y. R. Waghmare, Phys. Rev. C 2, 1191 (1970); K. Klingenbeck, W. Knüpfer, M. G. Huber, and P. W. M. Glaudemans, Phys. Rev. C 15, 1483 (1977).

[9] J. P. Elliott, A. D. Jackson, H. A. Mavromatis, E. A. Sanderson, and B. Singh, Nucl. Phys. A121, 241 (1968).

[10] F. Osterfeld, Rev. Mod. Phys. 64, 491 (1992); S. -O. Bäckman, G. E. Brown, and J. A. Niskanen, Phys. Rep. 124, 1 (1985).

[11] G. Bertsch, J. Borysowicz, H. McManus, and W. G. Love, Nucl. Phys. A284, 399 (1977).

[12] R. E. McDonald, J. A. Becker, R. A. Chalmers, and D. H. Wilkinson, Phys. Rev. C 10, 333 (1974).

[13] J. D. Walecka, in Muon Physics, edited by V. H. Hughes and C. S. Wu (Academic, New York, 1975), Vol. II; J. S. O'Connell, T. W. Donnelly, and J. D. Walecka, Phys. Rev. C 6, 719 (1972); T. W. Donnelly and J. D. Walecka, Nucl. Phys. A274, 368 (1976); T. W. Donnelly and W. C. Haxton, At. Data Nucl. Data Tables 23, 103 (1979).
[14] D. H. Wilkinson and B. E. F. Macefield, Nucl. Phys. A232, 58 (1974).

[15] OXBASH, the Oxford, Buenos-Aires, Michigan State, Shell Model Program, B. A. Brown, A. Etchegoyen, and W. D. M. Rae, MSU Cyclotron Laboratory Report No. 524, 1986 (unpublished).

[16] D. J. Millener and D. Kurath, Nucl. Phys. A255, 315 (1975).

[17] L. B. Auerbach et al. (LSND Collaborations), Phys. Rev. C 64, 065501 (2001).

[18] B. E. Bodmann et al. (KARMEN Collaboration), Phys. Lett. B332, 251 (1994).

[19] A. C. Hayes, P. Navrátil, and J. P. Vary, Phys. Rev. Lett. 91, 12502 (2003).

[20] E. Kolb, K. Langanke, and P. Vogel, Nucl. Phys. A652, 91 (1999).

[21] B. A. Armbruster et al. (KARMEN Collaboration), Phys. Lett. B423, 15 (1998).

[22] R. Maschuw, Prog. Part. Nucl. Phys. 40, 183 (1998).

[23] T. E. Drake, E. L. Tomusiak, and H. S. Caplan, Nucl. Phys. A118, 138 (1968); A. Yamaguchi, T. Terasawa, K. Nakahara, and Y. Torizuka, Phys. Rev. C 3,1750 (1971).

[24] X. Yang et al., Phys. Rev. C 48, 1158 (1993).

[25] C. Gaarde et al., Nucl. Phys. A422, 189 (1984).

[26] H. Okamura et al., Phys. Lett. B345, 1 (1995).

[27] J. Rapaport, T. Taddeucci, C. Gaarde, C. D. Goodman, C. C. Foster, C. A. Goulding, D. Horen, E. Sugarbaker, T. G. Masterson, and D. Lind, Phys. Rev. C 24, 335 (1981).

[28] J. R. Comfort, S. M. Austin, P. T. Debevec, G. L. Moake, R. W. Finlay, and W. G. Love, Phys. Rev. C 21, 2147 (1980). 
[29] T. Suzuki, H. Sagawa, and K. Hagino, Phys. Rev. C 68, 014317 (2003).

[30] R. E. Pywell, B. L. Berman, J. G. Woodworth, J. W. Jury, K. G. McNeill, and M. N. Thompson, Phys. Rev. C 32, 384 (1985); D. J. McLean, M. N. Thompson, D. Zubanov, K. G. McNeill, J. W. Jury, and B. L. Berman, ibid. 44, 1137 (1991).

[31] M. Th. Keil, G. G. Raffelt, and H. -Th. Janka, Astrophys. J. 590, 971 (2003).

[32] W. Hauser and H. Feshbach, Phys. Rev. 87, 366 (1952).

[33] R. L. Walter and P. P. Guss, in Proceedings of the International Conference on Nuclear Data for Basic and Applied Science, Santa Fe, May 13-17, 1985, p. 1079 (unpublished).

[34] V. Avrigeanu, P. E. Hodgson, and M. Avrigeanu, Phys. Rev. C 49, 2136 (1994).

[35] T. Belgya, O. Bersillon, R. Capote, T. Fukahori, G. Zhigang, S. Goriely, M. Herman, A. V. Ignatyuk, S. Kailas, A. Koning, P. Oblozhinsky, V. Plujko, and P. Young, Handbook for Calculations of Nuclear Reaction Data: Reference Input Parameter Library (IAEA, Vienna, 2006). Available online at http://wwwnds.iaea.org/RIPL-2/

[36] E. K. Warburton and B. A. Brown, Phys. Rev. C 46, 923 (1992).
[37] B. M. Preedom and B. H. Wildenthal, Phys. Rev. C 6, 1633 (1972).

[38] T. T. S. Kuo, Nucl. Phys. A103, 71 (1967).

[39] B. S. Pudliner, V. R. Pandharipande, J. Carlson, S. C. Pieper, and R. B. Wiringa, Phys. Rev. C 56, 1720 (1997).

[40] D. Gazit and N. Barnea, Phys. Rev. C 70, 048801 (2004).

[41] T. Yoshida, T. Kajino, H. Yokomakura, K. Kimura, A. Takamura, and D. H. Hartmann, Phys. Rev. Lett. 96, 091101 (2006).

[42] T. Yoshida, T. Kajino, and D. H. Hartmann, Phys. Rev. Lett. 94, 231101 (2005).

[43] T. Shigeyama and K. Nomoto, Astrophys. J. 360, 242 (1990).

[44] R. D. Hoffman and S. E. Woosley, Neutrino interaction cross sections and branching ratios, 1992, http://wwwphys.llnl.gov/Research/RRSN/nu_csbr/neu_rate.html

[45] T. Yoshida, M. Terasawa, T. Kajino, and K. Sumiyoshi, Astrophys. J. 600, 204 (2004).

[46] J. M. Lattimer and M. Prakash, Astrophys. J. 550, 426 (2001).

[47] T. Yoshida, T. Kajino, T. Suzuki, S. Chiba, T. Otsuka, A. Takamura, K. Kimura, H. Yokomakura, and D. H. Hartmann (in preparation). 